DOI: 10.2478/ausi-2014-0004

\title{
Nonexistence of a Kruskal-Katona type theorem for double-sided shadow minimization in the Boolean cube layer
}

\author{
Maksim BASHOV \\ Lomonosov Moscow State University \\ email: max.bashov@gmail.com
}

\begin{abstract}
A double-sided shadow minimization problem in the Boolean cube layer is investigated in this paper. The problem is to minimize the size of the union of the lower and upper shadows of a k-uniform family of subsets of [n]. It is shown that if $3 \leq k \leq n-3$, there is no total order such that all its initial segments have minimal double-sided shadow.
\end{abstract}

Denote by $\left(\begin{array}{c}{[n]} \\ k\end{array}\right)$ the family of all subsets of the set $[n]=\{1,2, \ldots, n\}$ having the size $k$. Let $\mathcal{F} \subseteq\left(\begin{array}{c}{[n]} \\ k\end{array}\right)$. The lower shadow $\Delta \mathcal{F}$ is the $(k-1)$-uniform family of sets $A$ such that there exists $B \in \mathcal{F}, A \subset B$. Similarly, the upper shadow $\nabla \mathcal{F}$ is the $(k+1)$-uniform family of sets $A$ such that there exists $B \in \mathcal{F}, A \supset B$. The double-sided shadow $\mathbf{\nabla F}$ is the union of the families $\Delta \mathcal{F}$ and $\nabla \mathcal{F}$.

A family $\mathcal{F} \subseteq\left(\begin{array}{c}{[n]} \\ k\end{array}\right)$ is minimal in terms of lower shadow (upper shadow, double-sided shadow) if $|\Delta \mathcal{F}| \leq|\Delta \mathcal{G}|$ (corr., $|\nabla \mathcal{F}| \leq|\nabla \mathcal{G}|,|\nabla \mathcal{F}| \leq|\nabla \mathcal{G}|$ ) for each family $\mathcal{G} \subseteq\left(\begin{array}{c}{[\mathrm{n}]} \\ k\end{array}\right)$ such that $|\mathcal{G}|=|\mathcal{F}|$.

A set $A$ lexicographically precedes a set $B(A<$ lex $B)$ if and only if $\max ((A \backslash$ B) $\cup(B \backslash A)) \in B$.

Kruskal [8] and Katona [7] described solutions of the single-sided shadow minimization problem:

Kruskal-Katona theorem. The initial lexicographical segments of $\left(\begin{array}{c}{[n]} \\ k\end{array}\right)$ are minimal families in terms of lower shadow.

A simple modern proof of this theorem is given in [1]. Clements and Lindström [6] generalized this result to the products of chains. Analogues of the

Computing Classification System 1998: G.2.1

Mathematics Subject Classification 2010: 05D05, 06A07, 68R15

Key words and phrases: shadow minimization, Boolean cube, double-sided shadow, ideal weight 
Kruskal-Katona theorem are also proved for a wide variety of structures, including the products of stars and their dual posets [5].

The Kruskal - Katona theorem describes minimal families in terms of a total order defined on $\left(\begin{array}{c}{[n]} \\ k\end{array}\right)$. In this paper we prove that it is impossible to describe the solutions of the double-sided minimization problem as initial segments of a total order.

We say that a total order defined on $\left(\begin{array}{c}{[n]} \\ k\end{array}\right)$ is minimizing if all its initial segments are minimal in terms of double-sided shadow. The following statement is proven in [2]:

Theorem 1 [2] If $\mathrm{k}=3$ and $\mathrm{n} \geqslant 8$, then there does not exist a minimizing total order on $\left(\begin{array}{c}{[n]} \\ k\end{array}\right)$. For every total order defined on $\left(\begin{array}{c}{[n]} \\ k\end{array}\right)$ there exists a number $\mathrm{m} \leqslant 4 \mathrm{n}-14$ such that the initial segment of the order having the length $\mathrm{m}$ is not minimal in terms of double-sided shadow.

Since $\left(\begin{array}{c}{[n]} \\ k\end{array}\right)$ is isomorphic to $\left(\begin{array}{c}{[n]} \\ n-k\end{array}\right)$, a similar result holds for $k=n-3$.

It is known [4] that the lexicographical order is minimizing when $k \geq n-2$, i. e., in this case families minimizing the lower shadow are also minimal in terms of double-sided shadow. Similarly, if $k \leq 2$, then the colexicographical order is minimizing the double-sided shadow.

Define a partial order induced by the standard shifting operator (see, e.g., [1]) on $\left(\begin{array}{c}{[n]} \\ k\end{array}\right)$. Let $A$ and $B$ be elements of $\left(\begin{array}{c}{[n]} \\ k\end{array}\right), A=\left\{a_{1}, a_{2}, \ldots, a_{k}\right\}, B=$ $\left\{b_{1}, b_{2}, \ldots, b_{k}\right\}$, and their elements are sorted in ascending order, i. e., $a_{1}<$ $a_{2}<\ldots<a_{k}, b_{1}<b_{2}<\ldots<b_{k}$. The set $A$ precedes the set $B(A \sqsubseteq B)$ if $a_{i} \leq b_{i}$ for each $i=1,2, \ldots, k$. A family $\mathcal{F}$ is an ideal if $A \in \mathcal{F}$ and $B \sqsubseteq A$ imply $\mathrm{B} \in \mathcal{F}$. By $\mathcal{I}(A)$ denote the minimal ideal containing $A$, i. e., the family $\left\{B \in\left(\begin{array}{c}{[n]} \\ k\end{array}\right) \mid B \sqsubseteq A\right\}$.

Theorem 2 [2] The family $\mathcal{C}_{1}(\mathrm{n}, \mathrm{k})=\mathcal{I}(\{2,3, \ldots, \mathrm{k}, \mathrm{n}\})$ is the unique minimal in terms of double-sided shadow ideal of $\left(\begin{array}{c}{[n]} \\ k\end{array}\right)$ having cardinality $1+\mathrm{k}(\mathrm{n}-\mathrm{k})$.

The following statement gives a simple additive formula for the size of an ideal's shadow.

Lemma 3 [2] If $\mathcal{F} \subseteq\left(\begin{array}{c}{[n]} \\ k\end{array}\right)$ is an ideal, then $|\nabla \mathcal{F}|=\sum_{A \in \mathcal{F}} s(A)$, where $s(A)=$ $s_{l}(A)+s_{\mathfrak{u}}(A), s_{l}(A)=\min ([n] \backslash A)-1, s_{\mathfrak{u}}(A)=n-\max A$.

The weight function $s$ is monotone with respect to the partial order $\sqsubseteq$ :

Lemma $4[2] A \sqsubseteq B$ implies $s(A) \geq s(B)$. 
Let $A=\left\{1,2, \ldots, m, a_{m+1}, \ldots, a_{k-1}, a_{k}\right\} \in\left(\begin{array}{c}{[n]} \\ k\end{array}\right), a_{m+1}>m+1$. Denote

$$
A^{0}=\left\{2,3, \ldots, m+1, a_{m+1}, \ldots, a_{k-1}, n\right\} .
$$

Put also $\widehat{\mathcal{I}}\left(A^{0}\right)=\left\{B \in\left(\begin{array}{c}{[n]} \\ k\end{array}\right), B^{0}=A^{0}\right\}$. Suppose $p=\min \left(\left\{q: a_{q}>q+1\right\} \cup\{k\}\right)$. The poset $\left(\widehat{\mathcal{I}}\left(A^{0}\right), \sqsubseteq\right)$ contains a least point

$$
\left\{1,2, \ldots, p-1, a_{p}, \ldots, a_{k-1}, a_{k-1}+1\right\}
$$

in case $p \neq k$ and a least point $\{1,2, \ldots, k\}$ in case $p=k$. Denote this least point by $A^{1}$.

By $\left.\mathcal{F}\right|_{\mathrm{q}}$ we denote the family $\{A \in \mathcal{F}, s(A)=\mathrm{q}\}$.

Lemma 5 [4] Let $\left.A^{0} \in\left(\begin{array}{c}{[n]} \\ k\end{array}\right)\right|_{0}, \mathcal{F}=\widehat{\mathcal{I}}\left(A^{0}\right) \backslash\left\{A^{1}\right\}$ if $A^{1}=\{1,2, \ldots, k\}$, and $\mathcal{F}=\widehat{\mathcal{I}}\left(\mathrm{A}^{0}\right)$ otherwise. Then $\mathcal{F}$ is isomorphic as a poset to the product of two chains having lengths $s_{\mathfrak{l}}\left(A^{1}\right)+1$ and $s_{\mathfrak{u}}\left(A^{1}\right)+1$, and the layers of the product correspond to the families $\left.\widehat{\mathcal{I}}\left(\mathrm{A}^{0}\right)\right|_{\mathrm{m}}$ under the isomorphism.

Lemma $6 \mathrm{~A}^{0} \sqsubseteq \mathrm{B}^{0}$ if and only if $\mathrm{A}^{1} \sqsubseteq \mathrm{B}^{1}$.

Proof. In case $A^{1}=\{1,2, \ldots, k\}$ the statement follows from the facts that $A^{0}=\{2,3, \ldots, k, n\}$ is the least set of the family $\left.\left(\begin{array}{c}{[n]} \\ k\end{array}\right)\right|_{0}$ in terms of the partial order $\sqsubseteq$, and $A^{1}$ is the least set of $\left(\begin{array}{c}{[n]} \\ k\end{array}\right)$.

Suppose

$$
\begin{aligned}
& A^{0}=\left\{2,3, \ldots, p, a_{p}, \ldots, a_{k-1}, n\right\}, a_{p}>p+1 \\
& B^{0}=\left\{2,3, \ldots, q, b_{q}, \ldots, b_{k-1}, n\right\}, b_{q}>q+1 .
\end{aligned}
$$

Then

$$
\begin{aligned}
& A^{1}=\left\{1,2, \ldots, p-1, a_{p}, \ldots, a_{k-1}, a_{k-1}+1\right\}, \\
& B^{1}=\left\{1,2, \ldots, q-1, b_{q}, \ldots, b_{k-1}, b_{k-1}+1\right\} .
\end{aligned}
$$

It remains to note that the condition $A^{0} \sqsubseteq B^{0}$ and the condition $A^{1} \sqsubseteq B^{1}$ are equivalent to $p \geq q, a_{j} \leq b_{j}$ if $p+1 \leq j \leq k-1$.

Suppose $A^{0}=\left\{2,3, \ldots, p, a_{p}, \ldots, a_{k-1}, n\right\}, a_{p}>p+1$. Note that if $A^{0} \neq$ $\{2,3, \ldots, k, n\}$, then the possible immediate successors of $A^{0}$ in terms of $\sqsubseteq$ are

$$
\begin{aligned}
A^{10} & =\left\{2,3, \ldots, p-1, p+1, a_{p}, \ldots, a_{k-2}, a_{k-1}, n\right\} \\
A^{u 0} & =\left\{2,3, \ldots, p-1, p, a_{p}, \ldots, a_{k-2}, a_{k-1}+1, n\right\}, \\
\tilde{A}_{j}^{0} & =\left\{2,3, \ldots, p-1, p, a_{p}, \ldots, a_{j}+1, \ldots, a_{k-2}, a_{k-1}, n\right\} .
\end{aligned}
$$


Also note that $s\left(A^{l 1}\right)=s\left(A^{u 1}\right)=s\left(A^{1}\right)-1$, and $s\left(\tilde{A}_{j}^{1}\right)=s\left(A^{1}\right)$.

Suppose $<_{\min }$ is a minimizing total order defined on $\left(\begin{array}{c}{[n]} \\ k\end{array}\right)$. The properties of the standard shifting operator [1] imply that without loss of generality we can suppose that the initial segments of $<_{\min }$ are ideals, i. e., $A \sqsubseteq B$ implies $A<_{\min } \mathrm{B}$.

By $\operatorname{ord}(A)$ denote the number of sets preceding $A$ in the order $<_{\min }$.

Lemma 7 Let $<_{\min }$ is a minimizing order on $\left(\begin{array}{c}{[n]} \\ k\end{array}\right), A<_{\min } \mathrm{B}<_{\min } \mathrm{C}$ and $\mathrm{A}^{0}=\mathrm{C}^{0}$. Then $\mathrm{A}^{0}=\mathrm{B}^{0}=\mathrm{C}^{0}$.

Proof. Suppose $B^{0} \neq A^{0}$.

Without loss of generality, $A=A^{1}, B=B^{1}$. Indeed, since $A^{1} \sqsubseteq A, B^{1} \sqsubseteq B$, it holds that $A^{1}<_{\min } A, B^{1}<_{\min } B$. If $A^{1}<_{\min } B^{1}$, we put $A^{\prime}=A^{1}, B^{\prime}=B^{1}$, $C^{\prime}=C$. If $A^{1}>_{\min } B^{1}$, we put $A^{\prime}=B^{1}, B^{\prime}=A^{1}, C^{\prime}=B$.

Without loss of generality, for all $D$ such that $D<_{\min } A, D^{0} \neq A^{0}$ it holds that $\mathrm{D}^{0}<_{\min } A$. If this property doesn't hold, we put $A^{\prime}=D^{1}, B^{\prime}=A$, $C^{\prime}=D^{0}$ and check the property again. This reassignment doesn't break the property from the previous paragraph, and an infinite chain of reassignments is impossible since $A^{\prime}<_{\min } A$ and the family $\left\{A^{\prime}: A^{\prime}<_{\min } A\right\}$ is finite.

We can assume that $B$ is the least set of the family $\left\{B: B^{0} \neq A^{0}, B>_{\min } A\right\}$ in terms of the order $<_{\min }$. Since the initial segments of $<_{\min }$ are ideals, by Lemma 6 the set $B^{0}$ is either incomparable to $A^{0}$ in the order $\sqsubseteq$, or is an immediate successor of $A^{0}$.

We can assume that $C$ is the least set of the family $\left\{C: C^{0}=A^{0}, C>_{\min } B\right\}$ in terms of the order $<_{\min }$.

Denote by $\mathcal{F}$ the initial segment of $<_{\text {min }}$ having the length ord(B). Since $<_{\min }$ is a minimizing order, and $\mathcal{F} \cup\{\mathrm{B}\}$ is its initial segment, it holds that $|\boldsymbol{Z}(\mathcal{F} \cup\{\mathrm{B}\})| \leq|\boldsymbol{Z}(\mathcal{F} \cup\{\mathrm{C}\})|$, therefore

$$
s(B) \leq s(C)
$$

Since $A \sqsubseteq C, A^{0}=C^{0}$, it follows from Lemma 5 that $s(C) \leq s(A)-1$.

Denote by $\mathcal{G}$ the initial segment of $<_{\min }$ having the length $\operatorname{ord}(A)$.

Suppose $A$ is incomparable to $B$ in the partial order $\sqsubseteq$. In this case the family $\mathcal{G} \cup\{B\}$ is an ideal, and by the definition of a minimizing order it holds that $|\boldsymbol{Z}(\mathcal{G} \cup\{A\})| \leq|\mathrm{Z}(\mathcal{G} \cup\{\mathrm{B}\})|$, therefore $s(A) \leq s(B)$. This implies $s(C)<s(B)$, a contradiction with (1).

Suppose $A$ is comparable to $B$ in the partial order $\sqsubseteq$. Note that $A^{0} \neq$ $\{2,3, \ldots, k, n\}$, because Theorem 2 implies that $\mathcal{C}_{1}(n, k)=\widehat{\mathcal{I}}(\{2,3, \ldots, k, n\})$ is an initial segment of $<_{\min }$. Then, since $A^{0}$ is an immediate predecessor of $B^{0}$ 
in the order $\sqsubseteq, s(B) \geq s(A)-1$. Therefore (1) implies $s(B)=s(A)-1$, and either $B^{0}=A^{10}$, or $B^{0}=A^{u 0}$.

Without loss of generality, $B^{0}=A^{u 0}$, that is

$$
\begin{aligned}
A & =\left\{1,2, \ldots, p, a_{p+1}, \ldots, a_{k-2}, q-1, q\right\}, \\
B & =\left\{1,2, \ldots, p, a_{p+1}, \ldots, a_{k-2}, q, q+1\right\}, a_{p+1}>p+2 .
\end{aligned}
$$

Since $A^{\prime}=\left\{1,2, \ldots, p, a_{p+1}, \ldots, a_{k-2}, q-1, q+1\right\} \sqsubseteq B$, it holds that $A^{\prime}<_{\text {min }}$ B. Note also that $\mathrm{q}+1 \leq \mathrm{n}$, and $\mathrm{n}-\mathrm{q} \geq 1$.

Consider the family $\mathcal{A}=\{A \cup\{\mathrm{r}\} \backslash\{\mathrm{q}\}, \mathrm{q}+1<\mathrm{r} \leq \mathrm{n}\}$. Note that for every $\mathrm{A}^{\prime \prime} \in \mathcal{A}$ it holds that $\mathrm{s}\left(\mathrm{A}^{\prime \prime}\right)<\mathrm{s}(\mathrm{A})-1=\mathrm{s}(\mathrm{B})$, and $\mathcal{A}$ is a chain in the partial order $\sqsubseteq$. Let us show that for every $A^{\prime \prime} \in \mathcal{A}$ it holds that $A^{\prime \prime}<_{\min } B$. Suppose $\mathrm{A}^{\prime \prime}$ is the least set in $\mathcal{A}$ such that $A^{\prime \prime}>_{\min } \mathrm{B}$. Then

$$
\left|\nabla\left(\mathcal{F} \cup\left\{A^{\prime \prime}\right\}\right)\right|=|\nabla \mathcal{F}|+s\left(A^{\prime \prime}\right)<|\nabla \mathcal{F}|+s(B)=|\nabla(\mathcal{F} \cup\{B\})|,
$$

a contradiction with the minimality of the family $\mathcal{F} \cup\{B\}$, which is an initial segment of $<_{\min }$.

Note that $C=A \cup\{p+1\} \backslash\{p\}$, since for every $A^{\prime \prime} \in \widehat{\mathcal{I}}\left(A^{0}\right)$ distinct from the sets $A, A^{\prime}$ and $A \cup\{p+1\} \backslash\{p\}$ it holds that $s\left(A^{\prime \prime}\right)<s(B)=s(C)$. Therefore $\operatorname{ord}(B)=\operatorname{ord}(A)+s_{\mathfrak{u}}(A)$.

Let us show that the immediate successors of $B$ in the order $<_{\min }$ are the sets $B \cup\{r\} \backslash\{q+1\}, q+1<r \leq n$. Suppose that the immediate successor of $(B \backslash\{q+1\}) \cup\{r\}$ is $D \neq B^{\prime}=B \cup\{r+1\} \backslash\{q+1\}$. The initial segments of $<_{\text {min }}$ having lengths ord(D) and ord(D) +1 are minimal in terms of double-sided shadow, and therefore

$$
s(D) \leq s(B \cup\{r+1\} \backslash\{q+1\})<s(C) .
$$

The families

$$
\mathcal{H}=\mathcal{F} \cup\{B\} \cup\{B \cup\{s\} \backslash\{q+1\}, q+1<s \leq r\}
$$

and $\mathcal{H} \cup\{\mathrm{D}\}$ are ideals, therefore $\mathrm{D} \in \widehat{\mathcal{I}}\left(\mathrm{A}^{0}\right)$ implies $\mathrm{D}=\mathrm{C}$, and $\mathrm{D} \in \widehat{\mathcal{I}}\left(\mathrm{B}^{0}\right)$ implies $D=B^{\prime}$. Hence, $D \notin \widehat{\mathcal{I}}\left(A^{0}\right) \cup \widehat{\mathcal{I}}\left(B^{0}\right)$. Since $D^{1}<_{\min } A, D^{0} \neq A^{0}$ implies $\mathrm{D}^{0}<_{\min } A$, it holds that $\mathrm{D}=\mathrm{D}^{1}$. If $\mathrm{D}$ is incomparable to $A$ in terms of $\sqsubseteq$, then it follows from (2) that

$$
|\nabla(\mathcal{G} \cup\{\mathrm{D}\})|=|\nabla \mathcal{G}|+s(\mathrm{D})<|\nabla \mathcal{G}|+s(C)<|\nabla \mathcal{G}|+s(A)=|\nabla(\mathcal{G} \cup\{A\})|,
$$

and that is a contradiction with the fact that $\mathcal{G} \cup\{\mathrm{A}\}$ is an initial segment $<_{\text {min }}$ and therefore is minimal in terms of double-sided shadow. If $\mathrm{D}$ is comparable 
to $A$ and incomparable to $B$, then, since $\mathcal{H}$ is an ideal, Lemma 6 implies that $D^{0}$ is an immediate successor of $A^{0}$ in the order $\sqsubseteq$, and $s(D) \geq s(A)-$ $1=s(C)$, a contradiction with (2). Hence, D is comparable to B. Since $D^{0}$ is an immediate successor of $\mathrm{B}^{0}$ in $\sqsubseteq$ and $s(\mathrm{D})<\mathrm{s}(\mathrm{C})$, it holds that $\mathrm{D}=$ $\left\{1,2, \ldots, p, a_{p+1}, \ldots, a_{k-2}, q+1, q+2\right\}$. Since $B \cup\{q+2\} \backslash\{q+1\} \sqsubseteq D$, it is true that $B \cup\{q+2\} \backslash\{q+1\}<_{\min } D$ and $r>q+2$. But then $s(B \cup\{r+1\} \backslash\{q+1\})<$ $s(\mathrm{D})$, a contradiction with (2).

Thus, $\mathcal{F}^{\prime}=\mathcal{F} \cup\{B\} \cup\{B \cup\{r\} \backslash\{q+1\}, q+1<r \leq n\}$ is an initial segment of $<_{\min }$. Denote by $D$ an immediate successor of $B \cup\{n\} \backslash\{q+1\}$ in terms of the order $<_{\min }$. Let us show that $s(D) \geq s(C)-1$. Indeed, if $D \in \widehat{\mathcal{I}}\left(A^{0}\right)$, then $\mathrm{D}=\mathrm{C}$, because $\mathcal{F} \cup\{\mathrm{D}\}$ is an ideal. Similarly, $\mathrm{D} \notin \widehat{\mathcal{I}}\left(\mathrm{B}^{0}\right)$. If $\mathrm{D} \notin \widehat{\mathcal{I}}\left(A^{0}\right) \cup \widehat{\mathcal{I}}\left(\mathrm{B}^{0}\right)$, then $s(D) \geq s(A)$ when $D$ is incomparable to $B$, and $s(D) \geq s(B)-1=s(C)-1$ in case $\mathrm{B} \sqsubseteq \mathrm{D}$. Therefore

$$
\begin{aligned}
& \left|\mathbf{z}\left(\mathcal{F}^{\prime} \cup\{D\}\right)\right|=|\boldsymbol{z} \mathcal{F}|+s(B)+\sum_{r=q+2}^{n} s(B \cup\{r\} \backslash\{q+1\})+s(D)= \\
& =|\boldsymbol{F}|+\sum_{r=0}^{n-q-1}(s(C)-r)+s(D) .
\end{aligned}
$$

Consider the family $\mathcal{F}^{\prime \prime}=\mathcal{F} \cup\{\mathrm{C}\} \cup\{\mathrm{C} \cup\{\mathbf{r}\} \backslash\{\mathbf{q}\}, \mathbf{r}<\mathbf{q} \leq \mathbf{n}\}$. Note that $\left|\mathcal{F}^{\prime \prime}\right|=\left|\mathcal{F}^{\prime} \cup\{\mathrm{D}\}\right|=|\mathcal{F}|+\mathrm{n}-\mathrm{q}$, and

$$
\begin{aligned}
\left|\nabla \mathcal{F}^{\prime \prime}\right| & =|\nabla \mathcal{F}|+s(C)+\sum_{r=q}^{n} s(C \cup\{r\} \backslash\{q\})= \\
& =|\nabla \mathcal{F}|+\sum_{r=0}^{n-q}(s(C)-r)=|z \mathcal{F}|+\sum_{r=0}^{n-q-1}(s(C)-r)+s(C)-(n-q) .
\end{aligned}
$$

If $n-q>2$, it follows that $\left|8 \mathcal{F}^{\prime \prime}\right|<\left|\nabla\left(\mathcal{F}^{\prime} \cup\{D\}\right)\right|$, a contradiction with the minimizing property of $<_{\min }$. If $n-q=1$, then there does not exist $D$ such that $\mathrm{B} \sqsubseteq \mathrm{D}, \mathrm{B}^{0} \neq \mathrm{D}^{0}$ and $\mathrm{s}(\mathrm{D})=\mathrm{s}(\mathrm{B})-1$, therefore $\mathrm{s}(\mathrm{D}) \geq \mathrm{s}(\mathrm{C})$, and again $\left|\nabla \mathcal{F}^{\prime \prime}\right|<\left|\boldsymbol{\nabla}\left(\mathcal{F}^{\prime} \cup\{\mathrm{D}\}\right)\right|$.

Corollary 8 Let $<$ be a total order defined on $\left(\begin{array}{c}{[n]} \\ k\end{array}\right)$ such that all its initial segments are ideals, $\mathrm{A}<\mathrm{B}<\mathrm{C}$ and $\mathrm{A}^{0}=\mathrm{C}^{0} \neq \mathrm{B}^{0}$. Then there exists a number $\mathrm{m}$ such that the initial segment of $<$ having the length $\mathrm{m}$ is not a minimal family in terms of double-sided shadow, and $m \leq \min \{\operatorname{ord}(B), \operatorname{ord}(A)+2 n\}$.

Thus, the families $\widehat{\mathcal{I}}\left(A^{0}\right)$ are segments of any minimizing total order. The following statement describes how the sets from these families are sorted by a minimizing order. 
Lemma 9 Suppose $<_{\min }$ is a minimizing order, $s(A)>0, s_{l}(A)=p, s_{\mathfrak{u}}(A)=$ $\mathrm{n}-\mathrm{q}$, and $\mathrm{B}$ is the immediate successor of $\mathrm{A}$ in $<_{\min }$. If $\mathrm{s}_{\mathrm{l}}\left(A^{1}\right)<\mathrm{s}_{\mathfrak{u}}\left(A^{1}\right)$, then

$$
B= \begin{cases}A \cup\{q+1\} \backslash\{q\}, & \text { if } q<n, \\ A \cup\left\{p+1, n-s_{\mathfrak{u}}\left(A^{1}\right)\right\} \backslash\{p, q\}, & \text { if } \mathbf{q}=n .\end{cases}
$$

If $s_{\mathfrak{l}}\left(A^{1}\right)>s_{\mathfrak{u}}\left(A^{1}\right)$, then

$$
B= \begin{cases}A \cup\{p+1\} \backslash\{p\}, & \text { if } p>0, \\ A \cup\{p+1, q+1\} \backslash\left\{s_{l}\left(A^{1}\right)+1, q\right\}, & \text { if } p=0 .\end{cases}
$$

And if $s_{l}\left(A^{1}\right)=s_{u}\left(A^{1}\right)$, then either for each $A \in \widehat{\mathcal{I}}\left(A^{0}\right)$ its immediate successor in $<_{\min }$ is defined by (3), or for each $A$ it is defined by (4).

Proof. This statement follows from Lemma 5, monotonicity and symmetry of the function s defined on $\widehat{\mathcal{I}}\left(A^{0}\right)$ and the two-dimensional case of the ClementsLindström theorem.

The order of families $\widehat{\mathcal{I}}\left(\mathrm{A}^{0}\right)$ in the minimizing order is described by the following two statements.

Lemma 10 Suppose $<_{\min }$ is a minimizing order, for each $\mathrm{C}^{0} \sqsubseteq \mathrm{A}^{0}$ it holds that $\mathrm{C}^{0}<_{\min } \mathrm{B}^{0}$, for each $\mathrm{C}^{0} \sqsubseteq \mathrm{B}^{0}$ it holds that $\mathrm{C}^{0}<_{\min } \mathrm{A}^{0}$, and $\mathrm{s}\left(\mathrm{A}^{1}\right)<$ $\mathrm{s}\left(\mathrm{B}^{1}\right)$. Then $\mathrm{A}^{1}<_{\min } \mathrm{B}^{1}$.

Proof. Assume the converse. Suppose $A^{0}$ and $B^{0}$ meet the conditions of the lemma, and $A^{1}>_{\min } B^{1}$. Denote by $\mathcal{F}$ the initial segment of $<_{\min }$ having the length ord $\left(B^{1}\right)$. Then $\mathcal{F} \cup\left\{A^{1}\right\}$ is an ideal, and since $A^{1} \notin \mathcal{F}$,

$$
\left|\mathbb{Z}\left(\mathcal{F} \cup\left\{A^{1}\right\}\right)\right|=|\mathbb{Z} \mathcal{F}|+s\left(A^{1}\right)<|\mathbb{F}|+s\left(B^{1}\right)=\left|\mathbb{Z}\left(\mathcal{F} \cup\left\{B^{1}\right\}\right)\right|,
$$

a contradiction to minimality of the initial segment $\mathcal{F} \cup\left\{B^{1}\right\}$.

Lemma 11 Suppose $<_{\min }$ is a minimizing order, for each $\mathrm{C}^{0} \sqsubseteq \mathrm{A}^{0}$ it holds that $\mathrm{C}^{0}<_{\min } \mathrm{B}^{0}$, for each $\mathrm{C}^{0} \sqsubseteq \mathrm{B}^{0}$ it holds that $\mathrm{C}^{0}<_{\min } \mathrm{A}^{0}$, and $\mathrm{s}\left(\mathrm{A}^{1}\right)=$ $s\left(B^{1}\right), \max \left\{s_{l}\left(A^{1}\right), s_{\mathfrak{u}}\left(A^{1}\right)\right\}>\max \left\{s_{l}\left(B^{1}\right), s_{\mathfrak{u}}\left(B^{1}\right)\right\}$. Then $A^{1}<_{\min } B^{1}$.

Proof. Assume the converse. Suppose $A^{0}$ and $B^{0}$ meet the conditions of the lemma, and $A^{1}>_{\min } B^{1}$. Denote by $\mathcal{F}$ the initial segment of $<_{\min }$ having the length ord $\left(B^{1}\right)$. Without loss of generality we can assume that $s_{\mathfrak{u}}\left(A^{1}\right)=$ 
$n-q^{\prime} \geq s_{l}\left(A^{1}\right)=p^{\prime}, s_{u}\left(B^{1}\right)=n-q^{\prime \prime} \geq s_{l}\left(B^{1}\right)=p^{\prime \prime}$. Then since $s_{l}\left(B^{1}\right)>$ $s_{l}\left(A^{1}\right) \geq 0$, Lemma 9 states that the family

$$
\mathcal{F}^{\prime}=\mathcal{F} \cup\left\{B^{1}\right\} \cup\left\{B^{1} \cup\{r\} \backslash\left\{q^{\prime \prime}\right\}, q^{\prime \prime}<r \leq n\right\} \cup\left\{B^{1} \cup\left\{p^{\prime \prime}+1\right\} \backslash\left\{p^{\prime \prime}\right\}\right\}
$$

is an initial segment of $<_{\min }$. Consider the family

$$
\mathcal{F}^{\prime \prime}=\mathcal{F} \cup\left\{A^{1}\right\} \cup\left\{A^{1} \cup\{r\} \backslash\left\{q^{\prime}\right\}, q^{\prime}<r \leq n+q^{\prime}+1-q^{\prime \prime}\right\} .
$$

Note that $\left|\mathcal{F}^{\prime}\right|=\left|\mathcal{F}^{\prime \prime}\right|=|\mathcal{F}|+n-q^{\prime \prime}+2$, and since $n-q^{\prime \prime}=s_{\mathfrak{u}}\left(B^{1}\right) \geq s_{l}\left(B^{1}\right) \geq 1$, it holds that

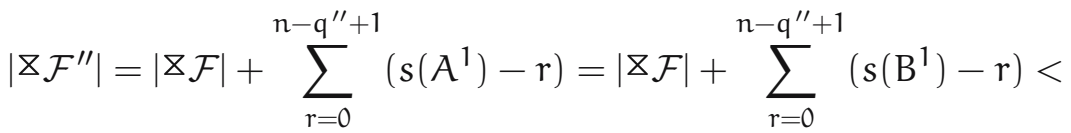

$$
\begin{aligned}
& <|\boldsymbol{F}|+\sum_{r=0}^{n-q^{\prime \prime}}\left(s\left(B^{1}\right)-r\right)+s\left(B^{1}\right)-1=\left|z \mathcal{F}^{\prime}\right|,
\end{aligned}
$$

a contradiction to the definition of a minimizing order.

Since the least set of $\left(\begin{array}{c}{[n]} \\ k\end{array}\right) \backslash \mathcal{C}_{1}(n, k)$ in terms of $\sqsubseteq$, namely,

$$
\{1,2, \ldots, k-2, k+1, k+2\}
$$

belongs to $\widehat{\mathcal{I}}(\{2,3, \ldots, k-1, k+1, n\})$, the families $\mathcal{C}_{1}(n, k)$ and $\widehat{\mathcal{C}}_{1}(n, k)=$ $\mathcal{C}_{1}(n, k) \cup \widehat{\mathcal{I}}(\{2,3, \ldots, k-1, k+1, n\})$ are initial segments of $<_{\text {min }}$. The family $\left(\begin{array}{c}{[n]} \\ k\end{array}\right) \backslash \widehat{\mathcal{C}}_{1}(n, k)$ contains two minimal sets. In case $n \neq 2 k$ we can infer which one is smaller in terms of $<_{\min }$.

Lemma 12 If $\mathrm{n}>2 \mathrm{k}, \mathrm{A} \in \widehat{\mathcal{I}}(\{2,3, \ldots, \mathrm{k}-1, \mathrm{k}+2, \mathrm{n}\}), \mathrm{B} \in \widehat{\mathcal{I}}(\{2,3, \ldots, \mathrm{k}-$ $2, k, k+1, n\})$, and $<_{\min }$ is a minimizing order, then $\mathrm{B}<_{\min } \mathrm{A}$.

Proof. Note that $A^{0}=\{2,3, \ldots, k-1, k+2, n\}$ and $B^{0}=\{2,3, \ldots, k-2, k, k+$ $1, n\}$ meet the conditions of Lemma 11 , and

$$
\max \left(s_{l}\left(A^{1}\right), s_{\mathfrak{u}}\left(A^{1}\right)\right)=n-k-2<n-k-1=\max \left(s_{l}\left(B^{1}\right), s_{\mathfrak{u}}\left(B^{1}\right)\right) .
$$

Theorem 13 If $4 \leqslant \mathrm{k}<\frac{\mathrm{n}}{2}$, then there does not exist a minimizing total order on $\left(\begin{array}{c}{[\mathrm{n}]} \\ k\end{array}\right)$. For every total order defined on $\left(\begin{array}{c}{[\mathrm{n}]} \\ k\end{array}\right)$ there exists a number $m \leqslant 1+k(n-k)+(k-1)(2 n-2 k-3)$ such that the initial segment of the order having the length $\mathrm{m}$ is not minimal in terms of double-sided shadow. 
Proof. Consider the initial segment $\mathcal{L}$ of the order $<_{\min }$ having the length

$$
|\mathcal{I}(\{2,3, \ldots, k-1, k+2, n\})|=1+k(n-k)+(k-1)(2 n-2 k-3) .
$$

It follows from Lemmas 7, 9, 10, 12 that this segment is the union of the ideal $\mathcal{I}(\{2,3, \ldots, k-2, k, k+1, n\})$ and the family of $n-2 k$ sets belonging to $\widehat{\mathcal{I}}(\{2,3, \ldots, k-3, k, k+1, k+2, n\})$. Counting the size of the double-sided shadow by Lemma 3 , we get

$$
|\nabla \mathcal{L}|=|8 \mathcal{I}(\{2,3, \ldots, k-1, k+2, n\})|+(n-2 k)(k-3),
$$

a contradiction to the definition of a minimizing order.

Since $\left(\begin{array}{c}{[n]} \\ k\end{array}\right)$ is isomorphic to $\left(\begin{array}{c}{[n]} \\ n-k\end{array}\right)$, the same result holds when $\frac{n}{2}<k \leq n-4$. It is known [3] that there is a nested system of minimal sets contained in $\mathcal{C}_{1}(n, k) \cup\{\{1,2, \ldots, k-2, k+1, k+2\}\}$, therefore it is impossible to get an estimate for $m$ lower than $2+k(n-k)$.

Now consider the case $n=2 k, k \geqslant 5$.

Theorem 14 If $5 \leqslant \mathrm{k}=\frac{\mathrm{n}}{2}$, then there does not exist a minimizing total order on $\left(\begin{array}{c}{[\mathrm{n}]} \\ k\end{array}\right)$. For every total order defined on $\left(\begin{array}{c}{[\mathrm{n}]} \\ k\end{array}\right)$ there exists a number $m \leqslant 1+k^{2}+(2 k-3)(k-2)+\frac{k(k-1)^{2}}{2}$ such that the initial segment of the order having the length $\mathrm{m}$ is not minimal in terms of double-sided shadow.

Proof. Consider the family

$$
\mathcal{F}=\mathcal{I}(\{3,4, \ldots, k+1, n\}) \cup \mathcal{I}(\{2,3, \ldots, k-2, k, k+2, n\}) .
$$

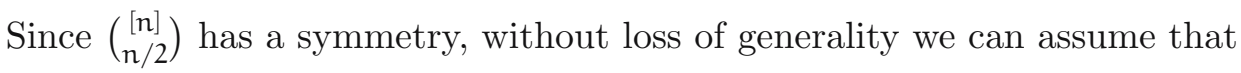

$$
\{2,3, \ldots, k-1, k+2, n\}>_{\min }\{2,3, \ldots, k-2, k, k+1, n\} .
$$

Lemmas 10 and 11 determine the subsequent order of the families $\widehat{\mathcal{I}}\left(A^{0}\right)$ in the minimizing order, and the initial segment $\mathcal{L}$ of $<_{\min }$ having the length $|\mathcal{F}|$ contains the ideals $\mathcal{I}(\{3,4, \ldots, k+1, n\}), \mathcal{I}(\{2,3, \ldots, k-1, k+3, n\})$ and the set $\{1,2, \ldots, k-2, k+4, k+5\}$. Counting the size of the shadow, we get $|\nabla \mathcal{L}|=|\nabla \mathcal{F}|+k-4$, a contradiction to the definition of a minimizing order.

Now we consider the cases not covered by Theorems 1, 13 and 14. There does not exist a minimizing order on $\left(\begin{array}{c}{[8]} \\ 4\end{array}\right)$, and the maximal size $m$ of a nested system of minimal families equals 42 , since the only minimal ideals having size 41 are $\mathcal{I}(\{2,4,5,8\}) \cup \mathcal{I}(\{2,3,7,8\})$ and $\mathcal{I}(\{2,3,6,8\}) \cup \mathcal{I}(\{3,4,5,8\})$, while 
the only minimal ideal having size 42 is $\mathcal{I}(\{2,4,6,8\})$. There does not exist a minimizing order on $\left(\begin{array}{c}{[7]} \\ 3\end{array}\right)$, and $m=15$, since the only minimal ideal having size 15 is $\mathcal{I}(\{1,6,7\})$, and it does not contain $\mathcal{C}_{1}(7,3)$. Finally, there is a minimizing order on $\left(\begin{array}{c}{[6]} \\ 3\end{array}\right)$ distinct from lexicographical:

$$
\begin{aligned}
& \{1,2,3\}<_{\min }\{1,2,4\}<_{\min }\{1,2,5\}<_{\min }\{1,2,6\}<_{\min }\{1,3,4\}<_{\min } \\
& \{1,3,5\}<_{\min }\{1,3,6\}<_{\min }\{2,3,4\}<_{\min }\{2,3,5\}<_{\min }\{2,3,6\}<_{\min } \\
& \{1,4,5\}<_{\min }\{1,4,6\}<_{\min }\{2,4,5\}<_{\min }\{2,4,6\}<_{\min }\{1,5,6\}<_{\min } \\
& \{2,5,6\}<_{\min }\{3,4,5\}<_{\min }\{3,4,6\}<_{\min }\{3,5,6\}<_{\min }\{4,5,6\} .
\end{aligned}
$$

Thus, a minimizing order exists on $\left(\begin{array}{c}{[n]} \\ k\end{array}\right)$ only in cases $k \leq 2, k \geq n-2$, and $\mathrm{n}=6, \mathrm{k}=3$.

\section{Acknowledgements}

The author would like to thank his advisor Prof. Alexander A. Sapozhenko for formulation of the problem and constant attention to the work. The author also thanks Dmitry V. Chistikov for useful discussions.

\section{References}

[1] R. Ahlswede, H. Aydinian, L. H. Khachatrian, More about shifting techniques, European J. Combin. 24, 5 (2003) 551-556. $\Rightarrow 53,54,56$

[2] M. A. Bashov, On minimisation of the double-sided shadow in the unit cube, Diskr. Mat., 23, 4 (2011) 115-132. $\Rightarrow 54$

[3] M. A. Bashov, Minimal families in terms of double-sided shadow in the Boolean cube layer, Electron. Notes in Discrete Math., 38 (2011) 117-122. $\Rightarrow 61$

[4] M. A. Bashov, Minimal in terms of double-sided shadow subsets of Boolean cube layer distinct from circles, Diskretn. Anal. Issled. Oper., 19, 5 (2012) 3-20. $\Rightarrow$ 54,55

[5] S. L. Bezrukov, U. Leck, Macaulay posets, Electron. J. Combin. (2004), Dynamic Survey DS12, http://www.combinatorics.org/. $\Rightarrow 54$

[6] G. F. Clements, B. Lindström, A generalization of a combinatorial theorem of Macaulay, J. Combin. Theory 7 (1969) 230-238. $\Rightarrow 53$

[7] G. O. H. Katona, A theorem of finite sets, Proc. Tihany Conference, New York, 1966, pp. 187-207. $\Rightarrow 53$

[8] J. Kruskal, The number of simplices in a complex. In: Mathematical Optimization Techniques, Berkeley, Los Angeles, Univ. of California Press, 1963, 251-278. $\Rightarrow$ 53

Received: February 5, 2013・Revised: May 31, 2013 